Pacific Journal of Mathematics

STABILITY OF UNFOLDINGS IN THE CONTEXT OF
EQUIVARIANT CONTACT-EQUIVALENCE

Ues GeRva 


\title{
STABILITY OF UNFOLDINGS IN THE CONTEXT OF EQUIVARIANT CONTACT-EQUIVALENCE
}

\author{
JeAn-JaCQues Gervais
}

\begin{abstract}
M. Golubitsky and D. Schaeffer introduced the notion of equivariant contact-equivalence between germs of $C^{\infty}$ equivariant mappings, in order to study perturbed bifurcation problems having a certain symmetry property. The main tool used is the so-called "Unfolding Theorem" for the qualitative description of the symmetry-preserving perturbations of these problems. From the point of view of applications, a relevant notion is that of stability of unfoldings. In this paper we prove the equivalence of the universality and the stability of unfoldings in the context of equivariant contact-equivalence.
\end{abstract}

1. Universal $\Gamma$-unfolding. Let $\Gamma$ be a compact Lie group acting orthogonally on $\mathbf{R}^{n}$ and $\mathbf{R}^{p}$. We write $\mathscr{E}_{n, p}$ for the space of $C^{\infty}$ germs $f:\left(\mathbf{R}^{n}, 0\right) \rightarrow \mathbf{R}^{p}$ of $\Gamma$-equivariant mappings (i.e. $f(\gamma x)=\gamma f(x)$ for all $\gamma \in \Gamma)$. The space of $\Gamma$-invariant $C^{\infty}$-germs $h:\left(\mathbf{R}^{n}, 0\right) \rightarrow \mathbf{R}$ (i.e. $h(\gamma x)=h(x)$ for all $\gamma \in \Gamma$ ) is denoted by $\mathscr{E}_{n} \Gamma$. In what follows we shall consider germs $G:\left(\mathbf{R}^{n} \times \mathbf{R}, 0\right) \rightarrow \mathbf{R}^{p}$ and $F:\left(\mathbf{R}^{n} \times \mathbf{R} \times \mathbf{R}^{q}, 0\right) \rightarrow \mathbf{R}^{p}$ and we shall assume that $\Gamma$ acts trivially on $\mathbf{R}$ and $\mathbf{R}^{q}$.

The notion of equivariant contact-equivalence introduced by Golubitsky and Schaeffer [3] is the following: if

Definition 1.1. We say that $G_{1}$ and $G_{2} \in \mathscr{E}_{n+1, p}^{\Gamma}$ are $\Gamma$-equivalent

$$
G_{1}(x, \lambda)=T(x, \lambda) G_{2}(X(x, \lambda), \Lambda(\lambda))
$$

where

$$
T:\left(\mathbf{R}^{n} \times \mathbf{R}, 0\right) \rightarrow \mathrm{Gl}_{p}(\mathbf{R}) \quad \text { is } C^{\infty} .
$$

$$
\begin{gathered}
(X, \Lambda):\left(\mathbf{R}^{n} \times \mathbf{R}, 0\right) \rightarrow\left(\mathbf{R}^{n} \times \mathbf{R}, 0\right) \quad \text { is } C^{\infty}, \\
\operatorname{det}\left(d_{x} X(0)\right)>0 \text { and } \Lambda^{\prime}(0)>0
\end{gathered}
$$

$$
X(\gamma x, \lambda)=\gamma X(x, \lambda) \quad \text { for all } \gamma \in \Gamma \text {. }
$$


A q-parameter $\Gamma$-unfolding of $G \in \mathscr{E}_{n+1, p}^{\Gamma}$ is a germ $F \in \mathscr{E}_{n+1+q, p}$ such that $F(x, \lambda, 0)=G(x, \lambda)$.

Definition 1.2. A $q$-parameter $\Gamma$-unfolding $F \in \mathscr{E}_{n+1+q, p}^{\Gamma}$ of $G \in$ $\mathscr{E}_{n+1, p}$ is said to be a universal $\Gamma$-unfolding if every $\Gamma$-unfolding $H$ of $G$ is induced by $F$ in the following way: assume that $H \in \mathscr{E}_{n+1+q^{\prime}, p}$; then there exist $C^{\infty}$ germs $T:\left(\mathbf{R}^{n} \times \mathbf{R} \times \mathbf{R}^{q^{\prime}}, 0\right) \rightarrow \mathrm{Gl}_{p}(\mathbf{R})$ and $(X, \Lambda, \alpha)$ : $\left(\mathbf{R}^{n} \times \mathbf{R} \times \mathbf{R}^{q^{\prime}}, 0\right) \rightarrow\left(\mathbf{R}^{n} \times \mathbf{R} \times \mathbf{R}^{q}, 0\right)$ such that:

$$
H(x, \lambda, \beta)=T(x, \lambda, \beta) \cdot F(X(x, \lambda, \beta), \Lambda(\lambda, \beta), \alpha(\beta)) .
$$

$$
X(\gamma x, \lambda, \beta)=\gamma X(x, \lambda, \beta) \quad \text { for all } \gamma \in \Gamma
$$

$$
\gamma^{-1} T(\gamma x, \lambda, \beta) \gamma=T(x, \lambda, \beta) \quad \text { for all } \gamma \in \Gamma \text {. }
$$

(1.2.5) $T(x, \lambda, 0) \equiv I_{p} \quad$ where $I_{p}$ is the identity $p \times p$-matrix.

Let $\mathscr{M}_{n+1, p}^{\Gamma}=\left\{T:\left(\mathbf{R}^{n+1}, 0\right) \rightarrow M_{p}(\mathbf{R}) \mid T\right.$ is $C^{\infty}$ and satisfies (1.2.3) $\}$ where $M_{p}(\mathbf{R})$ is the space of real $p \times p$ matrices. For $G \in \mathscr{E}_{n+1, p}$ we define

$$
\begin{aligned}
M_{G}: \mathscr{M}_{n+1, p}^{\Gamma} \oplus \mathscr{E}_{n+1, n}^{\Gamma} \rightarrow \mathscr{E}_{n+1, p}^{\Gamma} \\
(T, X) \mapsto T \cdot G+\left(d_{x} G\right) \cdot X
\end{aligned}
$$

and

$$
\begin{aligned}
N_{G}: \mathscr{E}_{1} & \rightarrow \mathscr{E}_{n+1, p}^{\Gamma} \\
\Lambda & \mapsto\left(d_{\lambda} G\right) \cdot \Lambda .
\end{aligned}
$$

Let

$$
\tilde{\Gamma} G=M_{G}\left(\mathscr{M}_{n+1, p}^{\Gamma} \oplus \mathscr{E}_{n+1, n}^{\gamma}\right) \quad \text { and } \quad \Gamma G=\tilde{\Gamma} G+N_{G}\left(\mathscr{E}_{1}\right)
$$

Roughly speaking, $\Gamma G$ is the tangent space to the orbit $O_{G}=\left\{G^{\prime} \in\right.$ $\mathscr{E}_{n+1, p} \mid G^{\prime}$ is $\Gamma$-equivalent to $\left.G\right\}$ at $G$. 
If $O_{G}$ has "finite codimension" that is $\operatorname{dim}_{\mathbf{R}} \mathscr{E}_{n+1, p} \Gamma \mid \Gamma G<\infty$ we have the unfolding theorem:

THEOREM 1.3 (Golubitsky-SCHAEFFER [3]). Let $G \in \mathscr{E}_{n+1, p}$ be of finite codimension and let $F \in \mathscr{E}_{n+1+q, p}$ be an unfolding of $G$. Then $F$ is a universal $\Gamma$-unfolding of $G$ if and only if

$$
\frac{\partial F}{\partial \alpha_{1}}(x, \lambda, 0), \ldots, \frac{\partial F}{\partial \alpha_{q}}(x, \lambda, 0)
$$

(where $\left.(x, \lambda, \alpha) \in \mathbf{R}^{n} \times \mathbf{R} \times \mathbf{R}^{q}\right)$ project onto a spanning set of $\mathscr{E}_{n+1, p} / \Gamma G$ i.e.

$$
\begin{aligned}
\mathscr{E}_{n+1, p}^{\Gamma}= & M_{G}\left(\mathscr{M}_{n+1, p}^{\Gamma} \oplus \mathscr{E}_{n+1, n}^{\Gamma}\right)+N_{G}\left(\mathscr{E}_{1}\right) \\
& +\mathbf{R}\left\{\frac{\partial F}{\partial \alpha_{i}}(x, \lambda, 0)\right\}
\end{aligned}
$$

REMARK 1.4. In fact, Golubitsky and Schaeffer [3] indicated how to prove the sufficiency of the condition (1.3.1). The necessity of (1.3.1) is proved in the following way (see [4] p. 259): Let $h \in \mathscr{E}_{n+1, p}$ and consider the one-parameter $\Gamma$-unfolding $H \in \mathscr{E}_{n+1+1, p}$ defined by $H(x, \lambda, t)=G(x, \lambda)+t h(x, \lambda)$. Since $F$ is universal, there exist $T, X, \Lambda$ and $\alpha$ as in 1.2 such that

$$
H(x, \lambda, t)=T(x, \lambda, t) \cdot F(X(x, \lambda, t), \Lambda(\lambda, t), \alpha(t)) .
$$

We obtain

$$
\begin{aligned}
h(x, \lambda) & =\left.\frac{\partial H}{\partial t}(x, \lambda, t)\right|_{t=0} \\
& =\left.\frac{\partial}{\partial t} T(x, \lambda, t) \cdot F(X(x, \lambda, t), \Lambda(\lambda, t), \alpha(t))\right|_{t=0}
\end{aligned}
$$

which is easily seen to belong to $\Gamma G+\mathbf{R}\left\{\partial F(x, \lambda, 0) / \partial \alpha_{i}\right\}$.

2. Stability of $\Gamma$-unfoldings. Let $U$ be a $\Gamma$-invariant open subset of $\mathbf{R}^{n} \times \mathbf{R} \times \mathbf{R}^{q}$. We write $C_{\Gamma}^{\infty}\left(U, \mathbf{R}^{p}\right)=\left\{F \in C^{\infty}\left(U, \mathbf{R}^{p}\right) \mid F(\gamma x, \lambda, \alpha)=\right.$ $\gamma F(x, \lambda, \alpha)$ for each $\gamma \in \Gamma\}$ endowed with the topology induced by the Whitney $C^{\infty}$-topology on $C^{\infty}\left(U, \mathbf{R}^{p}\right)$.

Definition 2.1. Let $U$ and $V$ be $\Gamma$-invariant open subsets of $\mathbf{R}^{n} \times$ $\mathbf{R} \times \mathbf{R}^{q}$. Let $\bar{F} \in C_{\Gamma}^{\infty}\left(U, \mathbf{R}^{p}\right)$ and let $\bar{H} \in C_{\Gamma}^{\infty}\left(V, \mathbf{R}^{p}\right)$. We say that $\bar{F}$, at $\left(x_{0}, \lambda_{0}, \alpha_{0}\right) \in U^{\Gamma}$ is $\Gamma$-equivalent to $\bar{H}$ at $\left(x_{1}, \lambda_{1}, \alpha_{1}\right) \in V^{\Gamma}$ if there exist 
$C^{\infty}$ germs

$$
\begin{aligned}
& T:\left(\mathbf{R}^{n} \times \mathbf{R} \times \mathbf{R}^{q},\left(x_{0}, \lambda_{0}, \alpha_{0}\right)\right) \longrightarrow \mathrm{Gl}_{p}(\mathbf{R}) \\
& X:\left(\mathbf{R}^{n} \times \mathbf{R} \times \mathbf{R}^{q},\left(x_{0}, \lambda_{0}, \alpha_{0}\right)\right) \longrightarrow\left(\mathbf{R}^{n}, x_{1}\right) \\
& \Lambda:\left(\mathbf{R} \times \mathbf{R}^{q},\left(\lambda_{0}, \alpha_{0}\right)\right) \longrightarrow\left(\mathbf{R}, \lambda_{1}\right) \\
& \phi:\left(\mathbf{R}^{q}, \alpha_{0}\right) \longrightarrow\left(\mathbf{R}^{q}, \alpha_{1}\right)
\end{aligned}
$$

such that

$$
F(x, \lambda, \alpha)=T(x, \lambda, \alpha) \cdot H(X(x, \lambda, \alpha), \Lambda(\lambda, \alpha), \phi(\alpha)),
$$

(2.1.3) $X(\gamma x, \lambda, \alpha)=\gamma X(x, \lambda, \alpha)$ and $\gamma^{-1} T(\gamma x, \lambda, \alpha) \gamma=T(x, \lambda, \alpha)$

for all $\gamma \in \Gamma$ where $U^{\Gamma}$ and $V^{\Gamma}$ are the sets of fixed points of $U$ and $V$ under the action of $\Gamma$.

Definition 2.2. Let $G \in \mathscr{E}_{n+1, p}$ and let $F \in \mathscr{E}_{n+1+q, p}$ be a $\Gamma$ unfolding of $G$. We say that $F$ is $\Gamma$-stable if, for every representative $\bar{F}$ of $F$ defined on an $\Gamma$-invariant open neighbourhood $U$ of $0 \in \mathbf{R}^{n} \times \mathbf{R} \times \mathbf{R}^{q}$, there is a neighbourhood $\mathscr{U}$ of $\bar{F}$ in $C_{\Gamma}^{\infty}\left(U, \mathbf{R}^{p}\right)$ such that, for every $\bar{H} \in \mathscr{U}$, there is a point $\left(x_{0}, \lambda_{0}, \alpha_{0}\right) \in U^{\Gamma}$ such that $\bar{F}$ at $(0,0,0)$ is $\Gamma$-equivalent to $\bar{H}$ at $\left(x_{0}, \lambda_{0}, \alpha_{0}\right)$.

The main result of this paper is:

THeOREM 2.3. Let $G \in \mathscr{E}_{n+1, p}$ be such that the $k$-jet $j^{k} G$ is $\Gamma$ sufficient. Then a $\Gamma$-unfolding $F \in \mathscr{E}_{n+1+q, p}$ of $G$ is universal if and only if it is $\Gamma$-stable.

Note. We say that the $k$-jet $j^{k} G$ of $G$ at 0 is $\Gamma$-sufficient if, for every $G_{1} \in \mathscr{E}_{n+1, p}^{\Gamma}$ such that $j^{k} G_{1}=j^{k} G, G$ and $G_{1}$ are $\Gamma$-equivalent in the sense of Definition 1.1.

Before proceeding to the proof of Theorem 2.3 we shall give some transversality properties of universal $\Gamma$-unfoldings.

3. Transversality. Let $J_{\Gamma}^{k}(n+1, p)=$ \{polynomial mappings on $\mathbf{R}^{n} \times \mathbf{R}$ into $\mathbf{R}^{p}$ which are $\Gamma$-equivariant and of degree $\left.\leq k\right\}$. This is the space of $k$-jets of the elements of $\mathscr{E}_{n+1, p}$ i.e.

$$
J_{\Gamma}^{k}(n+1, p)=\mathscr{E}_{n+1, p}^{\Gamma} /\left(\underline{m}_{x, \lambda}^{k+1} \cdot \mathscr{E}_{n+1, p}\right) \cap \mathscr{E}_{n+1, p}^{\Gamma}
$$

where $\underline{m}_{x, \lambda}$ is the maximal ideal of $\mathscr{E}_{n+1}=\mathscr{E}_{x, \lambda}$. Let

$$
\mathscr{G}^{k}=\left\{j^{k}(T, X, \Lambda) \mid T, X \text { and } \Lambda \text { are as in Definition } 1.1\right\} \text {. }
$$


Then $\mathscr{G}^{k}$ is an analytic Lie group which acts analytically on $J_{\Gamma}^{k}(n+1, p)$ in the following way: for $\theta \in \mathscr{G}^{k}$ and $z \in J_{\Gamma}^{k}(n+1, p)$, put $\theta z=j^{k}((T, X, \Lambda) \cdot G)$ where $\theta=j^{k}(T, X, \Lambda), z=j^{k} G$ and $((T, X, \Lambda) \cdot G)(x, \lambda)=T(x, \lambda) \cdot G(X(x, \lambda), \Lambda(\lambda))$. We shall write $O_{z}^{k}$ for the orbit of $z$ in $J_{\Gamma}^{k}(n+1, p)$ under the action of $\mathscr{G}^{k}$. As in [7, p. 41], we can prove

LEMMA 3.1. The tangent space to $O_{z}^{k}$ at $z$ is

$$
T_{z} O_{z}^{k}=\pi_{k}\left[M_{G}\left(\mathscr{M}_{n+1, p}^{\Gamma}+\left(\underline{m}_{x, \lambda} \cdot \mathscr{E}_{n+1, p}\right) \cap \mathscr{E}_{n+1, p}^{\Gamma}\right)+N_{G}\left(\mathscr{E}_{1}\right)\right]
$$

where $\pi_{k}: \mathscr{E}_{n+1, p}^{\Gamma} \rightarrow J_{\Gamma}^{k}(n+1, p)$ is the natural projection.

An immediate consequence (see e.g. [1]) is

Proposition 3.2. Let $G \in \mathscr{E}_{n+1, p}{ }_{\text {be }}$ buch that $j^{k} G$ is $\Gamma$-sufficient. Then

$$
\Gamma G \supset\left(\underline{m}_{x, \lambda}^{k+1} \cdot \mathscr{E}_{n+1, p}\right) \cap \mathscr{E}_{n+1, p}^{\Gamma}
$$

3.3. For $\bar{F} \in C_{\Gamma}^{\infty}\left(U, \mathbf{R}^{p}\right)$ and $(x, \lambda, \alpha) \in U$ we define the germ

$$
\begin{aligned}
F_{(x, \lambda)}^{\alpha}: & \left(\mathbf{R}^{n} \times \mathbf{R}, 0\right) \rightarrow \mathbf{R}^{p} \\
(y, \mu) & \mapsto \bar{F}(x+y, \lambda+\mu, \alpha)
\end{aligned}
$$

and we define

$$
\begin{gathered}
j_{*}^{k} \bar{F}: U \rightarrow \mathbf{R}^{n} \times \mathbf{R} \times J^{k}(n+1, p) \\
(x, \lambda, \alpha) \mapsto\left(x, \lambda, j^{k} F_{(x, \lambda)}^{\alpha}\right)
\end{gathered}
$$

where $J^{k}(n+1, p)$ is the space of $k$-jets of the elements of $\mathscr{E}_{n+1, p}$.

For $G \in \mathscr{E}_{n+1, p}^{\Gamma}$ we write $S_{z}^{k}$ for the submanifold of $J^{k}(n+1, p)$ equal to $\left(\mathbf{R}^{n+1}\right)^{\Gamma} \times O_{z}^{k} \times\left(J_{\Gamma}^{k}(n+1, p)\right)^{\perp}$, where $z=j^{k} G,\left(\mathbf{R}^{n+1}\right)^{\Gamma}$ is the set of fixed points under the action of $\Gamma$ and $\left(J_{\Gamma}^{k}(n+1, p)\right)^{\perp}$ is the orthogonal complement in $J^{k}(n+1, p)$ of the subspace $J_{\Gamma}^{k}(n+1, p)$.

LemMA 3.3. Let $F \in \mathscr{E}_{n+1+q, p}$ be a $\Gamma$-unfolding of $G \in \mathscr{E}_{n+1, p}$. Then $j_{*}^{k} F$ is transverse to $S_{z}^{k}$ at $(0,0,0)$ if and only if

(3.3.1) $\Gamma G+\mathbf{R}\left\{\frac{\partial F}{\partial \alpha_{i}}(x, \lambda, 0)\right\}+\left(\underline{m}_{x, \lambda}^{k+1} \cdot \mathscr{E}_{n+1, p}\right) \cap \mathscr{E}_{n+1, p}=\mathscr{E}_{n+1, p}$.

Proof. The range of $d\left(j_{*}^{k} F\right)_{(0,0)}$ is

$$
\mathbf{R}^{n} \times \mathbf{R} \times \pi_{k}\left[\mathbf{R}\left\{\frac{\partial F}{\partial x_{i}}(x, \lambda, 0), \frac{\partial F}{\partial \lambda}(x, \lambda, 0), \frac{\partial F}{\partial \alpha_{j}}(x, \lambda, 0)\right\}\right] .
$$


Hence the above transversality condition is satisfied if and only if

$$
\begin{aligned}
& \text { Range } d\left(j_{*}^{k} F\right)_{(0,0,0)}+T_{(0,0)}\left(\mathbf{R}^{n+1}\right)^{\Gamma} \times\{0\}+\{0\} \times T_{z} O_{z}^{k} \\
& \quad+\{0\} \times\left(J_{\Gamma}^{k}(n+1, p)^{\perp}\right. \\
& =\mathbf{R}^{n+1} \times J^{k}(n+1, p)
\end{aligned}
$$

hence, by virtue of Lemma 3.1,

$$
\pi_{k}\left[\Gamma G+\mathbf{R}\left\{\frac{\partial F}{\partial \alpha_{i}}(x, \lambda, 0)\right\}\right]+\left(J_{\Gamma}^{k}(n+1, p)\right)^{\perp}=J^{k}(n+1, p) .
$$

But

$$
\pi_{k}\left[\Gamma G+\mathbf{R}\left\{\frac{\partial F}{\partial \alpha_{i}}(x, \lambda, 0)\right\}\right] \subset J_{\Gamma}^{k}(n+1, p),
$$

and the desired result follows.

4. Proof of Theorem 2.3. Let $G \in \mathscr{E}_{n+1, p}$ be such that $z=j^{k} G$ is $\Gamma$-sufficient and let $F \in \mathscr{E}_{n+1+q, p}$ be a $\Gamma$-unfolding of $G$.

4.1. Universality $\Rightarrow$ stability. Suppose that $F$ is universal and let $\bar{F} \in C_{\Gamma}^{\infty}\left(U, \mathbf{R}^{p}\right)$ be a representative of $F$ on an open $\Gamma$-invariant neighbourhood of $0 \in \mathbf{R}^{n} \times \mathbf{R} \times \mathbf{R}^{q}$. From the unfolding theorem and Lemma 3.3, we conclude that $j_{*}^{k} F$ is transverse to $S_{z}^{k}$ at $(0,0,0)$. The Transversality Theorem (see [8, p. 321]) implies the existence of a neighbourhood $\mathscr{U}$ of $\bar{F}$ in $C^{\infty}\left(U, \mathbf{R}^{p}\right)$ such that, for every $\bar{H} \in \mathscr{U}, j_{*}^{k} \bar{H}$ intersects $S_{z}^{k}$ transversally at at least one point $\left(x_{0}, \lambda_{0}, \alpha_{0}\right) \in U$. Put $\mathscr{U}_{\Gamma}=\mathscr{U} \cap C_{\Gamma}^{\infty}\left(U, \mathbf{R}^{p}\right)$. Then for each $\bar{H} \in \mathscr{U}_{\Gamma}$, there exists $\left(x_{0}, \lambda_{0}, \alpha_{0}\right) \in$ $U$ such that $j_{*}^{k} \bar{H}\left(x_{0}, \lambda_{0}, \alpha_{0}\right) \in S_{z}^{k}$ and $j_{*}^{k} \bar{H}$ is transverse to $S_{z}^{k}$ at $\left(x_{0}, \lambda_{0}, \alpha_{0}\right)$. We shall show that $\bar{F}$, at $(0,0,0)$, is $\Gamma$-equivalent to $\bar{H}$ at $\left(x_{0}, \lambda_{0}, \alpha_{0}\right)$. Let $H$ be the germ at $(0,0,0)$ defined by $H(x, \lambda, \alpha)=$ $\bar{H}\left(x_{0}+x, \lambda_{0}+\lambda, \alpha_{0}+\alpha\right)$ and let $h$ be the germ at $(0,0) \in \mathbf{R}^{n} \times \mathbf{R}$ given by $h(x, \lambda)=\bar{H}\left(x_{0}+x, \lambda_{0}+\lambda, \alpha_{0}\right)$; since $j_{*}^{k} \bar{H}\left(x_{0}, \lambda_{0}, \alpha_{0}\right) \in S_{z}^{k}$, we have $\left(x_{0}, \lambda_{0}\right) \in\left(\mathbf{R}^{n+1}\right)^{\Gamma}$ and we deduce that $h \in \mathscr{E}_{n+1, p}^{\Gamma}$ since

$$
\begin{aligned}
h(\gamma x, \lambda) & =\bar{H}\left(x_{0}+\gamma x, \lambda_{0}+\lambda, \alpha_{0}\right) \\
& =\bar{H}\left(\gamma x_{0}+\gamma x, \lambda_{0}+\lambda, \alpha\right)=\gamma \bar{H}\left(x_{0}+x, \lambda_{0}+\lambda, \alpha_{0}\right)=\gamma h(x, \lambda)
\end{aligned}
$$

because $\bar{H} \in C_{\Gamma}^{\infty}\left(U, \mathbf{R}^{p}\right)$. Therefore $z_{0}=j^{k} h \in O_{z}^{k}$; hence $z_{0}$ is $\Gamma$ sufficient since $z$ is $\Gamma$-sufficient. Proposition 3.2 implies that

$$
\Gamma h \supset\left(\underline{m}_{x, \lambda}^{k+1} \cdot \mathscr{E}_{n+1, p}\right) \cap \mathscr{E}_{n+1, p}^{\Gamma} .
$$

On the other hand $O_{z}^{k}=O_{z_{0}}^{k}$, and so $j_{*}^{k} H$ is transverse at $(0,0,0)$ to 
$S_{z_{0}}^{k}$, and this is equivalent, by virtue of Lemma 3.3, to the equality

$$
\Gamma h+\mathbf{R}\left\{\frac{\partial H}{\partial \alpha_{j}}(x, \lambda, 0)\right\}+\left(\underline{m}_{x, \lambda}^{k+1} \cdot \mathscr{E}_{n+1, p}\right) \cap \mathscr{E}_{n+1, p}=\mathscr{E}_{n+1, p} \Gamma
$$

From this equality and (4.1.1) we deduce that

$$
\Gamma h+\mathbf{R}\left\{\frac{\partial H}{\partial \alpha_{j}}(x, \lambda, 0)\right\}=\mathscr{E}_{n+1, p} \Gamma
$$

and so, the unfolding theorem implies that $H$ is a universal $\Gamma$-unfolding of $h$.

The germs $h$ and $G$ are $\Gamma$-equivalent (as in Definition 1.1) since the jets $z=j^{k} G$ and $z_{0}=j^{k} h$ are $\Gamma$-sufficient and $O_{z}^{k}=O_{z_{0}}^{k}$. Thus, there exist $T, X$ and $\Lambda$ as in 1.1 such that

$$
h(x, \lambda)=T(x, \lambda) G(X(x, \lambda), \Lambda(\lambda)) .
$$

Put $\tilde{F}(x, \lambda, \alpha)=T(x, \lambda) F(X(x, \lambda), \Lambda(\lambda), \alpha)$; then

$$
\begin{aligned}
\tilde{F}(x, \lambda, 0) & =T(x, \lambda) \cdot F(X(x, \lambda), \Lambda(\lambda), 0) \\
& =T(x, \lambda) \cdot G(X(x, \lambda), \Lambda(\lambda))=h(x, \lambda),
\end{aligned}
$$

that is, $\tilde{F}$ is a $q$-parameter $\Gamma$-unfolding of $h$. But $H$ is universal $\Gamma$ unfolding; we then easily deduce that $H$ at $(0,0,0)$ is $\Gamma$-equivalent to $\tilde{F}$ at $(0,0,0)$. From there it is not difficult to see that $\bar{H}$ at $\left(x_{0}, \lambda_{0}, \alpha_{0}\right)$ is $\Gamma$-equivalent to $F$ at $(0,0,0)$ (see e.g. [2, p. 173]).

4.2. Stability $\Rightarrow$ universality. Suppose that $F$ is $\Gamma$-stable but is not universal which, by virtue of the unfolding theorem, is equivalent to

$$
\Gamma G+\mathbf{R}\left\{\frac{\partial F}{\partial \alpha_{i}}(x, \lambda, 0)\right\} \varsubsetneqq \mathscr{E}_{n+1, p} .
$$

Since $j^{k} G$ is $\Gamma$-sufficient we have $\Gamma G \supset\left(\underline{m}_{x, \lambda}^{k+1} \mathscr{E}_{n+1, p}\right) \cap \mathscr{E}_{n+1, p}$, and so (4.2.1) is equivalent to

$$
\Gamma G+\mathbf{R}\left\{\frac{\partial F}{\partial \alpha_{j}}(x, \lambda, 0)\right\}+\left(\underline{m}_{x, \lambda}^{k+1} \cdot \mathscr{E}_{n+1, p}\right) \cap \mathscr{E}_{n+1, p} \varsubsetneqq \mathscr{E}_{n+1, p}^{\Gamma} ;
$$

hence Lemma 3.3 implies that $j_{*}^{k} F$ is not transverse to $S_{z}^{k}$ at $(0,0,0)$.

We shall use the same method as S. Izumiya [5, p. 41]. By virtue of the foregoing there exists $w \in J^{k}(n+1, p)$ such that

$$
w \notin \text { Range } d\left(j_{*}^{k} F\right)_{(0,0,0)}+T_{(0,0, z)} S_{z}^{k} \text {. }
$$

We may assume that $w \in J_{\Gamma}^{k}(n+1, p)$ and thus $w \notin T_{z} O_{z}^{k}$. Let $U$ be a $\Gamma$-invariant neighbourhood of $(0,0,0)$ in $\mathbf{R}^{n} \times \mathbf{R} \times \mathbf{R}^{q}$ and let $\bar{F} \in C_{\Gamma}^{\infty}\left(U, \mathbf{R}^{p}\right)$ and $\bar{w}$, defined on $U \cap \mathbf{R}^{n} \times \mathbf{R} \times\{0\}$, be representatives of $F$ and $w$. For $t \in \mathbf{R}$, put $\bar{H}(x, \lambda, \alpha, t)=\bar{F}(x, \lambda, \alpha)+t \bar{w}(x, \lambda)$. Since $F$ 
is $\Gamma$-stable, there is $\varepsilon>0$ such that, for every $t_{0} \in[-\varepsilon, \varepsilon]$, there exists $\left(x_{0}, \lambda_{0}, \alpha_{0}\right) \in U^{\Gamma}$ such that $\bar{H}_{t_{0}}$ at $\left(x_{0}, \lambda_{0}, \alpha_{0}\right)$ is $\Gamma$-equivalent to $F$ at $(0,0,0)$, where $\bar{H}_{t_{0}}(x, \lambda, \alpha)=\bar{H}\left(x, \lambda, \alpha, t_{0}\right)$. In particular,

$$
\operatorname{dim} \operatorname{Range} d\left(j_{*}^{k} \bar{H}_{t_{0}}\right)_{\left(x_{0}, \lambda_{0}, \alpha_{0}\right)}=\operatorname{dim} \operatorname{Range} d\left(j_{*}^{k} F\right)_{(0,0,0)} \text {. }
$$

On the other hand,

$$
\text { dim Range } d\left(j_{*}^{k} \bar{H}\right)_{(0,0,0,0)}>\operatorname{dim} \text { Range } d\left(j_{*}^{k} F\right)_{(0,0,0)} .
$$

One easily sees (cf. [5, p. 41]) that there exists a submanifold $\Sigma$ of $J^{k}(n+1, p)$ such that $\Sigma$ contains a neighbourhood of $z$ in $O_{z}^{k}, \operatorname{cod} \Sigma=$ dim Range $d\left(j_{*}^{k} \bar{H}\right)_{(0,0,0,0)}$, and $j_{*}^{k} \bar{H}$ is transverse to $\Sigma$ at each point of $U \times[-\varepsilon, \varepsilon]$. But from Sard's Theorem it follows (see e.g. [6, p. 134]) that there exists $t_{0} \in[-\varepsilon, \varepsilon]$ such that $j_{*}^{k} \bar{H}_{t_{0}}$ is transverse to $\Sigma$ at every point of $U$. But, if $\varepsilon$ is small enough, there exists $\left(x_{0}, \lambda_{0}, \alpha_{0}\right) \in U^{\Gamma}$ such that $\bar{H}_{t_{0}}$ at $\left(x_{0}, \lambda_{0}, \alpha_{0}\right)$ is $\Gamma$-equivalent to $\bar{F}$ at $(0,0,0)$. Thus $j_{*}^{k} \bar{H}_{t_{0}}\left(x_{0}, \lambda_{0}, \alpha_{0}\right) \in\left\{\left(x_{0}, \lambda_{0}\right)\right\} \times O_{z}^{k} \subset S_{z}^{k}$; we therefore have the equality (4.2.2). On the other hand, since $j_{*}^{k} H_{t_{0}}$ intersects $\Sigma$ transversally at $\left(x_{0}, \lambda_{0}, \alpha_{0}\right)$ and $\operatorname{cod} \Sigma=\operatorname{dim} \operatorname{Range} d\left(j_{*}^{k} \bar{H}\right)_{(0,0,0,0)}$ we have

$$
\begin{aligned}
\operatorname{dim} \text { Range } d\left(j_{*}^{k} \bar{H}\right)_{(0,0,0,0)} & =\operatorname{dim} \text { Range } d\left(j_{*}^{k} \bar{H}_{t_{0}}\right)_{\left(x_{0}, \lambda_{0}, \alpha_{0}\right)} \\
& =\operatorname{dim} \text { Range } d\left(j_{*}^{k} \bar{F}\right)_{(0,0,0)}
\end{aligned}
$$

in contradiction with (4.2.3).

REMARK. As in the nonsymmetric context, one can consider the bifurcation parameter $\lambda$ to be multi-dimensional and proves analogous results (see [2]).

\section{REFERENCES}

[1] J. J. Gervais, Sufficiency of jets, Pacific J. Math., 72 (1977), 419-422.

[2] D Déformations $G$-verselles et $G$-stables, Canad. J. Math., XXXVI, no. 1 (1984), 9-21.

[3] M. Golubitsky and D. Schaeffer, Imperfect bifurcation in presence of symmetry, Comm. Math. Phys., 67 (1979), 205-232.

[4] Singularities and Groups in Bifurcation Theory I, Applied Math. Science, 51 (Springer-Verlag, New York, 1985).

[5] S. Izumiya, Stability of G-unfoldings, Hokkaido Math. J., 9 (1980), 36-45.

[6] J. C. Tougeron, Idéaux de Fonctions Différentiables, Ergebnisse Band 71 (SpringerVerlag, New York, 1972).

[7] G. Wasserman, Stability of Unfoldings, Lecture Notes 393, (Springer-Verlag, New York, 1974). 
[8] C. Zeeman, The Classification of Elementary Catastrophes of Codimension $\leq 5$, Lecture Notes 525 (Springer-Verlag, New York, 1976), 263-327.

Received September 12, 1985. This work was partly supported by a grant from NSERC of Canada and a "subvention FCAC" from the Department of Education of Québec.

UNIVERSITÉ LAVAL

QuÉbec, CaNada G1K 7P4 



\title{
PACIFIC JOURNAL OF MATHEMATICS EDITORS
}

\author{
V. S. VARADARAJAN \\ (Managing Editor) \\ University of California \\ Los Angeles, CA 90024 \\ HERBERT Clemens \\ University of Utah \\ Salt Lake City, UT 84112 \\ R. FINN \\ Stanford University \\ Stanford, CA 94305
}

\author{
HERMANN FLASCHKA \\ University of Arizona \\ Tucson, AZ 85721
}

Ramesh A. Gangolli University of Washington Seattle, WA 98195

VAUGHAN F. R. JONES University of California Berkeley, CA 94720
ROBION KIRBY

University of California

Berkeley, CA 94720

C. C. MOORE

University of California

Berkeley, CA 94720

HAROLD STARK

University of California, San Diego La Jolla, CA 92093

\section{ASSOCIATE EDITORS}

\author{
R. ARENS \\ E. F. BECKENBACH \\ B. H. NEUMANN \\ F. WOLF \\ K. YOSHIDA \\ (1906-1982)

\section{SUPPORTING INSTITUTIONS}

\section{UNIVERSITY OF ARIZONA} \\ UNIVERSITY OF BRITISH COLUMBIA \\ CALIFORNIA INSTITUTE OF TECHNOLOGY \\ UNIVERSITY OF CALIFORNIA \\ MONTANA STATE UNIVERSITY \\ UNIVERSITY OF NEVADA, RENO \\ NEW MEXICO STATE UNIVERSITY \\ OREGON STATE UNIVERSITY \\ UNIVERSITY OF OREGON \\ UNIVERSITY OF SOUTHERN CALIFORNIA \\ STANFORD UNIVERSITY \\ UNIVERSITY OF HAWAII \\ UNIVERSITY OF TOKYO \\ UNIVERSITY OF UTAH \\ WASHINGTON STATE UNIVERSITY \\ UNIVERSITY OF WASHINGTON
}

The Supporting Institutions listed above contribute to the cost of publication of this Journal, but they are not owners or publishers and have no responsibility for its content or policies.

Mathematical papers intended for publication in the Pacific Journal of Mathematics should be in typed form or offset-reproduced (not dittoed), double spaced with large margins. Please do not use built up fractions in the text of the manuscript. However, you may use them in the displayed equations. Underline Greek letters in red, German in green, and script in blue. The first paragraph must be capable of being used separately as a synopsis of the entire paper. In particular it should contain no bibliographic references. Please propose a heading for the odd numbered pages of less than 35 characters. Manuscripts, in triplicate, may be sent to any one of the editors. Please classify according to the scheme of Math. Reviews, Index to Vol. 39. Supply name and address of author to whom proofs should be sent. All other communications should be addressed to the managing editor, or Elaine Barth, University of California, Los Angeles, California 90024

There are page-charges associated with articles appearing in the Pacific Journal of Mathematics. These charges are expected to be paid by the author's University, Government Agency or Company. If the author or authors do not have access to such Institutional support these charges are waived. Single authors will receive 50 free reprints; joint authors will receive a total of 100 free reprints. Additional copies may be obtained at cost in multiples of 50 .

The Pacific Journal of Mathematics is issued monthly as of January 1966. Regular subscription rate: $\$ 190.00$ a year (5 Vols., 10 issues). Special rate: $\$ 95.00$ a year to individual members of supporting institutions.

Subscriptions, orders for numbers issued in the last three calendar years, and changes of address should be sent to Pacific Journal of Mathematics, P.O. Box 969, Carmel Valley, CA 93924, U.S.A. Old back numbers obtainable from Kraus Periodicals Co., Route 100, Millwood, NY 10546.

The Pacific Journal of Mathematics at P.O. Box 969, Carmel Valley, CA 93924 (ISSN 0030-8730) publishes 5 volumes per year. Application to mail at Second-class postage rates is pending at Carmel Valley, California, and additional mailing offices. Postmaster: send address changes to Pacific Journal of Mathematics, P.O. Box 969, Carmel Valley, CA 93924.

PUBLISHED BY PACIFIC JOURNAL OF MATHEMATICS, A NON-PROFIT CORPORATION Copyright (C) 1988 by Pacific Journal of Mathematics 


\section{Pacific Journal of Mathematics}

\section{Vol. 132, No. $2 \quad$ February, 1988}

Jeffery Marc Bergen and Luisa Carini, A note on derivations with power central values on a Lie ideal ..............................209

Alfonso Castro and Sumalee Unsurangsie, A semilinear wave equation

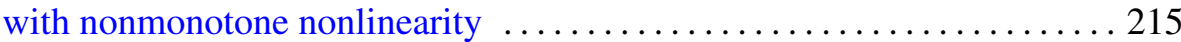

Marius Dadarlat, On homomorphisms of matrix algebras of continuous

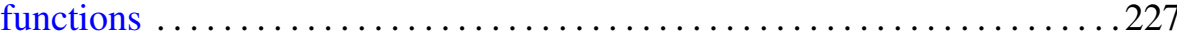

A. Didierjean, Quelques classes de cobordisme non orienté refusant de se

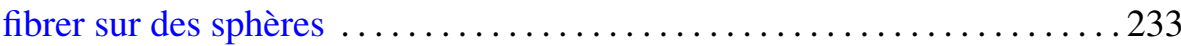

Edward George Effros and Zhong-Jin Ruan, On matricially normed spaces

Sherif El-Helaly and Taqdir Husain, Orthogonal bases are Schauder bases

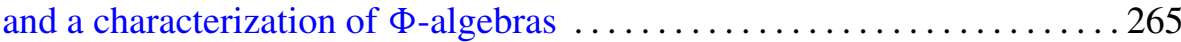

Edward Richard Fadell and Peter N-S Wong, On deforming $G$-maps to be

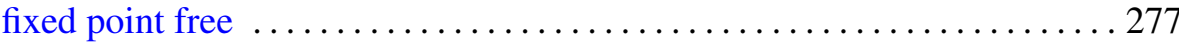

Jean-Jacques Gervais, Stability of unfoldings in the context of equivariant

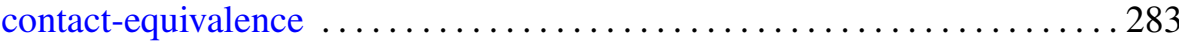

Douglas Martin Grenier, Fundamental domains for the general linear group

Ronald Scott Irving and Brad Shelton, Loewy series and simple projective

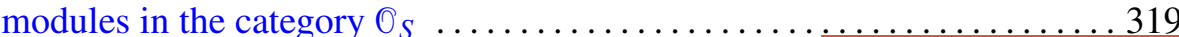

Russell Allan Johnson, On the Sato-Segal-Wilson solutions of the K-dV equation

Thomas Alan Keagy and William F. Ford, Acceleration by subsequence transformations

Min Ho Lee, Mixed cusp forms and holomorphic forms on elliptic varieties

Charles Livingston, Indecomposable surfaces in 4-space 